\title{
Floquet theory for seasonal environmental forcing of spatially explicit waterborne epidemics
}

\author{
Lorenzo Mari • Renato Casagrandi • Enrico Bertuzzo • \\ Andrea Rinaldo • Marino Gatto
}

Received: 5 November 2013 / Accepted: 26 March 2014 / Published online: 13 June 2014

(C) Springer Science+Business Media Dordrecht 2014

\begin{abstract}
The transmission of waterborne pathogens is a complex process that is heavily linked to the spatial characteristics of the underlying environmental matrix as well as to the temporal variability of the relevant hydroclimatological drivers. In this work, we propose a timevarying, spatially explicit network model for the dynamics of waterborne diseases. Applying Floquet theory, which allows to extend results of local stability analysis to periodic dynamical systems, we find conditions for pathogen invasion and establishment in systems characterized by fluctuating environmental forcing, thus extending to timevarying contexts the generalized reproduction numbers recently obtained for spatially explicit epidemiology of waterborne disease. We show that temporal variability may have multifaceted effects on the invasion threshold, as it can either favor pathogen invasion or make it less likely. Moreover, environmental fluctuations characterized by distinctive geographical signatures can produce diversified, highly nontrivial effects on pathogen invasion. Our study is complemented by numerical simulations, which show
\end{abstract}

Electronic supplementary material The online version of this article (doi: 10.1007/s12080-014-0223-y) contains supplementary material, which is available to authorized users.

L. Mari $(\bowtie) \cdot$ R. Casagrandi $\cdot$ M. Gatto

Dipartimento di Elettronica, Informazione e Bioingegneria, Politecnico di Milano, 20133 Milan, Italy

e-mail: lorenzo.mari@polimi.it

L. Mari · E. Bertuzzo · A. Rinaldo

Laboratory of Ecohydrology ECHO/IIE/ENAC,

École Polytechnique Fédérale de Lausanne, 1015 Lausanne, Switzerland

\footnotetext{
A. Rinaldo

Dipartimento di Ingegneria Civile, Edile ed Ambientale, Università di Padova, 35131 Padua, Italy
}

that pathogen establishment is neither necessary nor sufficient for large epidemic outbreaks to occur in time-varying environments. Finally, we show that our framework can be used to reliably characterize the early geography of epidemic outbreaks triggered by fluctuating environmental conditions.

Keywords Bifurcation analysis · Disease-free equilibrium $\cdot$ Monodromy matrix $\cdot$ Periodic signal . Environmental drivers $\cdot$ Invasion conditions

\section{Introduction}

Waterborne diseases are transmitted through the ingestion of pathogenic microorganisms (protozoa, bacteria, or viruses) that can be found in contaminated water or food. Diarrhea, commonly associated with waterborne pathogens, is responsible for the deaths of about 1.8 million people every year, thus representing one of the leading causes of death worldwide, especially among infants and children in low-income countries. According to the World Health Organization, most of that burden is attributable to unsafe water supply, lack of sanitation, and poor hygienic conditions (World Health Organization 2008).

The transmission of waterborne diseases is heavily linked to environmental drivers and their seasonal patterns (e.g., Altizer et al. 2006; McMichael et al. 2006; Eisenberg et al. 2007). This relation has been widely studied for cholera, probably the best known among potentially lethal waterborne diseases. The seasonality and interannual variability of endemic and epidemic cholera and the interplay between environmental drivers (such as temperature, precipitation, plankton concentration) and disease dynamics are in fact receiving increasing attention (e.g., Colwell 1996; Pascual 
et al. 2000, 2002, 2008; Bouma and Pascual 2001; Lipp et al. 2002; Ruiz-Moreno et al. 2007; Hashizume et al. 2008; de Magny et al. 2008; Mendelsohn and Dawson 2008; Akanda et al. 2009; Emch et al. 2010; Gaudart et al. 2013; Eisenberg et al. 2013a). However, although convincing empirical evidence shows that cholera occurrence is typically seasonal because of climatic and environmental factors, there is no clear indication yet of a robust, unambiguous correlation between a specific exogenous driver and the observed patterns of cholera incidence. Seasonal fluctuations are also often related to spatial variation of disease distribution-yet, despite its importance, seasonality has seldom been considered in spatially explicit models for waterborne disease dynamics. Some exceptions do exist, though. For instance, Bertuzzo et al. (2008) considered seasonal temperature patterns in a spatial simulation model of the cholera epidemic that stroke KwaZulu-Natal (South Africa) in 2000-2002; also, temperature and discharge fluctuations proved instrumental to explaining spatiotemporal patterns of cholera incidence in the Bengal region (Bertuzzo et al. 2012); seasonal precipitation patterns have been implied in the resurgence of cholera in Haiti (Rinaldo et al. 2012; Righetto et al. 2013); at a smaller spatial scale, Reiner et al. (2012) used a spatial stochastic model to show that localized sensitivity to climate forcing drives the endemic dynamics of cholera in the city of Dhaka, Bangladesh.

In general, defining the environmental conditions that can favor waterborne pathogen invasion and ensuing longterm persistence (endemism) still represents an open theoretical challenge, whose solution could bear major implications for health care practice. In highly idealized settings (i.e., disregarding both spatial dynamics and seasonal environmental fluctuations), threshold conditions for pathogen invasion and establishment are linked to the so-called basic reproduction number $R_{0}\left(R_{0}>1\right.$ being the necessary condition for pathogen invasion). For waterborne diseases (and in particular for cholera), expressions for $R_{0}$ have been proposed since the very first modeling studies (e.g., Capasso and Paveri-Fontana 1979; Codeço 2001; see also Tien and Earn 2010; Tian and Wang 2011). Attempts to define invasion thresholds for waterborne pathogens in more realistic cases are very recent. Gatto et al. $(2012,2013)$ analyzed a spatially explicit model for waterborne diseases and generalized the concept of basic reproduction number to spatially structured environments (see also Eisenberg et al. 2013b). They found that the condition that all the local basic reproduction numbers be larger than one is neither necessary nor sufficient for pathogen invasion to occur whenever the characteristic timescales of the epidemiological dynamics are shorter than those of pathogen relocation mechanisms (namely hydrological transport and human mobility), hence in cases in which spatial dynamics must be accounted for (Bertuzzo et al. 2010).
Regarding the interplay between temporal variability and pathogen invasion conditions, the definition of a reproduction number in periodic environments has been proposed by Bacaër and Guernaoui (2006) and Bacaër (2007) with application to vectorborne diseases. This definition has been subsequently extended to generic compartmental models by Wang and Zhao (2008), who provided a generalization of the so-called next-generation matrix method (see Diekmann et al. 1990, 2010; Diekmann and Heesterbeek 2000; van den Driessche and Watmough 2002) to periodically varying environments. The compartmental approach used by Wang and Zhao (2008) can be naturally extended to patchy environments (see also Zhang and Zhao 2007). Following the approach developed by Bacaër (2007) and by Wang and Zhao (2008), Zhou and Cui (2013) have studied invasion conditions in a spatially implicit model for cholera epidemics with periodic transmission rates. Using the same theoretical framework, Sardar et al. (2013) have recently estimated local reproduction numbers for cholera in Zimbabwe subject to seasonal environmental fluctuations. Long-term cholera dynamics in a time-varying (yet, again, spatially implicit) setting has been analyzed also via bifurcation analysis (Righetto et al. 2012). To the best of our knowledge, though, no attempts have been made so far to find waterborne pathogen invasion thresholds in a realistic setting, that is both spatially explicit and time-varying. It is also important to remark that in periodic environments sizable epidemic outbreaks may occur (because of transient dynamics) even if pathogen invasion conditions are not met-in other words, if long-term pathogen establishment is not possible (Bacaër and Gomes 2009).

In this work, we determine the conditions under which pathogen invasion is possible within a specific territory, described as a river basin, that is (i) endowed with certain demographic, epidemiological, climatic, and socioeconomic characteristics and (ii) subject to seasonal variations of the relevant environmental drivers. To that end, we make use of Floquet theory, a mathematical framework that allows the study of systems of periodically forced differential (or difference) equations (see Bittanti and Colaneri 2009, for a review of the theory and the related methods). In a nutshell, Floquet theory provides means to extend the results of local stability analysis to periodic dynamical systems. Despite its usefulness in studying and understanding nonequilibrium dynamics, it is seldom applied in epidemiological and ecological research (see Heesterbeek and Roberts 1995a, b for the first epidemiological applications and Klausmeier 2008 for a review of some ecological problems that can be addressed by the theory). Another important aim of our work is the analysis of the geography of epidemic outbreaks in time-varying environments, i.e., the prediction of the areas of the territory under study that will be initially hit should an epidemic outbreak unfold. This is especially 
important because knowledge of the epidemic epicenter can assist in an efficient allocation of interventions that can minimize the extent of a disease outbreak.

The paper is organized as follows. In "The model" we describe our spatially explicit, time-varying model for the dynamics of waterborne infections. In "Derivation of pathogen invasion conditions" we derive the condition for pathogen invasion. Then, in "Analysis of the invasion conditions: local model" and "Analysis of the invasion conditions: network model" the invasion condition is analyzed with respect to the parameters of the model, respectively in spatially implicit and explicit settings. The analysis of the invasion thresholds is complemented with numerical simulations of the network model to investigate transient epidemiological dynamics. "Spatial patterns of epidemic spread" is devoted to linking invasion conditions to the spatial patterns of epidemic outbreaks. Spatial analyses are pursued via both stability analysis (through Floquet theory) and numerical simulations. Finally, concluding remarks are given in "Discussion and conclusions".

\section{The model}

We generalize to time-varying environments a recent network model of waterborne disease dynamics that has already been used in both theoretical (Mari et al. 2012b; Gatto et al. 2013) and applied (Gatto et al. 2012; Mari et al. 2012a; Rinaldo et al. 2012; Righetto et al. 2013) studies. The model describes local epidemiological, demographic, and ecological dynamics, pathogen transport along water systems, and the effects of short-term human mobility on disease propagation. Network nodes represent human communities (villages, towns, or cities) of assigned population, arranged in a given spatial setting, and connected by hydrological pathways and human mobility (multilayer network model, see, e.g., Mari et al. 2011, 2014 for other ecological applications). Specifically, we assume here that human communities constitute the nodes of a so-called optimal channel network (OCN), i.e., a mathematical structure characterized by scaling forms that closely conform to the observed geomorphological features of real river networks (Rinaldo et al. 1992, 2014; Rodriguez-Iturbe et al. 1992). The OCN is embedded in a square of arbitrary side 1 .

Let $S_{i}(t)$ and $I_{i}(t)$ be the local abundances of susceptible and infected individuals in each node $i$ of the network at time $t$, and let $B_{i}(t)$ be the concentration of the pathogens (e.g., bacteria, viruses, or protozoa) in the local water reservoirs. Epidemiological dynamics and pathogen transport over the hydrological and human mobility networks can be described by the following set of $3 n$ ordinary differential equations:

$$
\begin{aligned}
& \frac{d S_{i}}{d t}=\mu\left(H_{i}-S_{i}\right)-\left\{\left[1-m_{i}(t)\right] \beta_{i}(t) \frac{B_{i}}{K+B_{i}}+m_{i}(t) \sum_{j=1}^{n} Q_{i j}(t) \beta_{j}(t) \frac{B_{j}}{K+B_{j}}\right\} S_{i} \\
& \frac{d I_{i}}{d t}=\left\{\left[1-m_{i}(t)\right] \beta_{i}(t) \frac{B_{i}}{K+B_{i}}+m_{i}(t) \sum_{j=1}^{n} Q_{i j}(t) \beta_{j}(t) \frac{B_{j}}{K+B_{j}}\right\} S_{i}-(\gamma+\mu+\alpha) I_{i} \\
& \frac{d B_{i}}{d t}=-\left[\mu_{B i}(t)+l_{i}(t)\right] B_{i}+\sum_{j=1}^{n} l_{j}(t) P_{j i}(t) \frac{W_{j}(t)}{W_{i}(t)} B_{j}+\frac{p_{i}(t)}{W_{i}(t)}\left\{\left[1-m_{i}(t)\right] I_{i}+\sum_{j=1}^{n} m_{j}(t) Q_{j i}(t) I_{j}\right\} .
\end{aligned}
$$

Model (1) represents a broad scheme of waterborne disease transmission across spatial and temporal scales. A comprehensive list of all model parameters is reported in Table 1 for easy reference. Note that some of them may be assumed to be time-dependent and/or site-specific, while others may be seen as constant in time and/or independent of the node.

Regarding local processes (Fig. 1a), the evolution of the susceptible compartment (first equation of model 1) is a balance between population demography and infections due to contact with the pathogen. The host population, if uninfected, is assumed to be at demographic equilibrium $H_{i}$, with $\mu$ being the human mortality rate. The parameter $\beta_{i}(t)$ represents the site-specific rate of exposure to contaminated water, and $B_{i} /\left(K+B_{i}\right)$ is the dose-response function describing the probability of becoming infected due to the exposure to a concentration $B_{i}$ of pathogens, with $K$ being the half-saturation constant (Codeço 2001). The dynamics of the infected compartment (second equation of model 1) is a balance between newly infected individuals and losses due to recovery or natural/pathogen-induced mortality, with $\gamma$ and $\alpha$ being the rates of recovery and mortality due to the disease, respectively. The evolution of the local concentration of pathogens that live free in the aquatic environment (third equation of model 1) assumes that pathogens are released in the water (e.g., excreted) by infected individuals 
Table 1 Model parameters and their definitions. Parameters for which dependence on node $i$ and/or time $t$ is not made explicit are assumed to be constant in space and/or time

\begin{tabular}{ll}
\hline Parameter & Definition \\
\hline$\mu$ & Baseline human mortality rate \\
$K$ & Half-saturation constant \\
$\gamma$ & Recovery rate from disease \\
$\alpha$ & Disease-related mortality rate \\
$H_{i}$ & Population size \\
$\beta_{i}(t)$ & Exposure rate \\
$p_{i}(t)$ & Contamination rate \\
$\mu_{B i}(t)$ & Pathogen mortality rate \\
$W_{i}(t)$ & Water reservoir volume \\
$l_{i}(t)$ & Pathogen transport rate \\
$P_{i j}(t)$ & Fraction of pathogens moving from node $i$ to node $j$ \\
$m_{i}(t)$ & Fraction of people moving from their home node \\
$Q_{i j}(t)$ & Fraction of people traveling from node $i$ to node $j$ \\
$n$ & Number of nodes in the network \\
\hline
\end{tabular}

and immediately diluted in a well-mixed local water reservoir of volume $W_{i}(t)$ at a rate $p_{i}(t)$. Free-living pathogens are also assumed to die at rate $\mu_{B i}(t)$.

To mimic hydrological transport (Fig. 1b), which defines the first layer of spatial connectivity, the spread of pathogens over the river network is described as a biased random walk process on an oriented graph (Bertuzzo et al. 2007, 2008). Specifically, we assume that pathogens can move between any two nodes $i$ and $j$ of the hydrological network at a rate $l_{i}(t)$ with a probability $P_{i j}(t)$. The rate $l_{i}(t)$ depends on both downstream advection and other possible pathogen transport mechanisms along the hydrological network, e.g., pathogen attachment to phyto- and zooplankton. The hydrological connection matrix $\mathbf{P}(t)=\left[P_{i j}(t)\right]$ is given by
$P_{i j}(t)=\left\{\begin{array}{ccc}P_{i}^{d}(t) & \text { if } & i \rightarrow j \\ P_{i}^{u}(t) & \text { if } & i \leftarrow j \\ 0 & \text { if } & i \leftrightarrow j,\end{array}\right.$

where $P_{i}^{d}(t)\left[P_{i}^{u}(t)\right]$ is the site-dependent fraction of pathogens moving along an outward (downstream) [inward (upstream)] edge (arrows indicate downstream connections). The transport process is assumed to be possibly nonconservative, i.e., $\sum_{j \in N_{i}} P_{i j}(t)=1-a_{i}(t)$, where $N_{i}$ is the set of neighbors connected to node $i$ (of cardinality $n_{i}^{d}+n_{i}^{u}$, where $n_{i}^{d}\left[n_{i}^{u}\right]$ is the outdegree [indegree] of node $i$, that is the number of outward [inward] edges) and $a_{i}(t) \geq 0$ is the node-dependent fraction of dispersing pathogens that do not reach any other node of the river network. This parameter can be used to prescribe specific boundary conditions (BCs) and/or other possible losses in the stream. To close the specification of $P_{i j}(t)$, we define the bias $b_{i}(t)$ of hydrological transport as the difference between downstream $\left(P_{i}^{d}(t)\right)$ and upstream $\left(P_{i}^{u}(t)\right)$ movement probabilities (path-wise partitioning sensu Johnson et al. 1995). Therefore, in the inner nodes of the network, where $n_{i}^{d}>0$ and $n_{i}^{u}>0$, the following equalities must hold:

$$
\begin{aligned}
& n_{i}^{d} P_{i}^{d}(t)+n_{i}^{u} P_{i}^{u}(t)=1-a_{i}(t) \\
& P_{i}^{d}(t)-P_{i}^{u}(t)=b_{i}(t),
\end{aligned}
$$

from which we get

$$
P_{i j}(t)=\left\{\begin{array}{ccc}
\frac{1+b_{i}(t) n_{i}^{u}-a_{i}(t)}{n_{i}^{d}+n_{i}^{u}} & \text { if } & i \rightarrow j \\
\frac{1-b_{i}(t) n_{i}^{d}-a_{i}(t)}{n_{i}^{d}+n_{i}^{u}} & \text { if } & i \leftarrow j \\
0 & \text { if } & i \leftrightarrow j .
\end{array}\right.
$$

As stated above, the hydrological connection matrix $\mathbf{P}(t)$ can account for proper BCs for the headwaters and the outlet of the river network. All the numerical results described later in the paper refer to reflecting $\mathrm{BCs}$ for the leaves, absorbing BCs for the outlet of the river network and conservative transport elsewhere. Therefore, we set $P_{u v}=1$ in

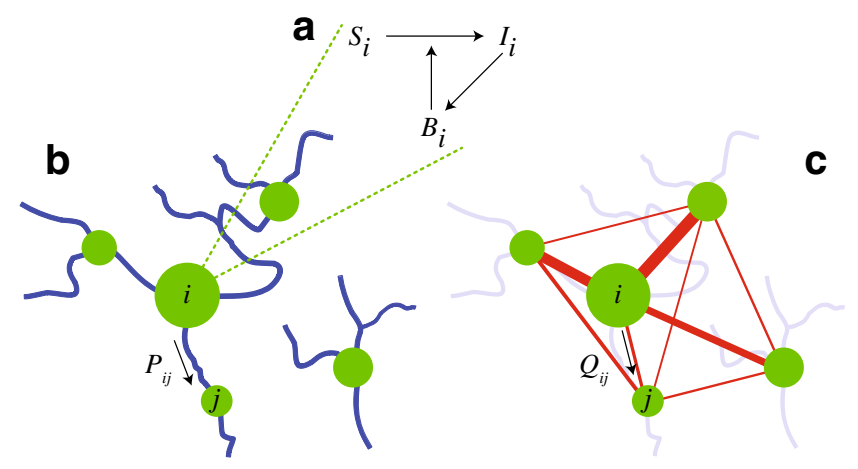

Fig. 1 A spatially explicit network model for the dynamics of waterborne diseases. a Local epidemiological dynamics in the $i$-th community. b Pathogen transport along the river network. c Human mobility network. Communities of different sizes constitute the nodes of the network model (green circles). Connections between nodes (specified by matrices $\mathbf{P}$ and $\mathbf{Q}$ ) can be due to hydrological pathways and/or human displacements 
every leaf $u$ of the network ( $v$ being its downstream nearest neighbor), $a_{i}(t)>0$ at the network outlet (conventionally labeled as node 1), and $a_{i}(t)=0$ in all the other nodes. Specifically, the fraction of particles absorbed at the outlet is assumed to be equal to the fraction that would move along a downstream edge, i.e., $a_{1}(t)=\left[1+b_{i}(t) n_{1}^{u}\right] /\left(1+n_{1}^{u}\right)$.

To describe human mobility (Fig. 1c), representing the second layer of spatial connectivity, we assume that susceptible and infected individuals can undertake short-term trips from the communities where they live toward other nodes. While traveling or commuting, susceptible individuals can be exposed to pathogens and return as infected carriers to the community where they usually live. Similarly, infected hosts can disseminate pathogens away from their home community. Human mobility patterns are defined according to a connection matrix $\mathbf{Q}(t)=\left[Q_{i j}(t)\right]$ in which individuals leave their original node (say $i$ ) with probability $m_{i}(t)$, reach their target location (say $j$ ) with probability $Q_{i j}(t)$ and then come back to node $i$. Matrix $\mathbf{Q}(t)$ is defined according to a gravity model (Erlander and Stewart 1990) in which

$$
Q_{i j}(t)=\frac{H_{j} \exp \left(-\frac{d_{i j}}{D_{i}(t)}\right)}{\sum_{k \neq i}^{n} H_{k} \exp \left(-\frac{d_{i k}}{D_{i}(t)}\right)},
$$

where $d_{i j}$ is the pairwise distance between any two nodes $i$ and $j$, and $D_{i}(t)$ is the scale parameter of the exponential kernel. Note that here, human mobility is assumed to be conservative, i.e., $\sum_{j} Q_{i j}(t)=1$, but different assumptions can be made as well. Gravity-like models, although very simple, have often been used in the epidemiological literature to describe human mobility (see Truscott and Ferguson 2012, for a recent discussion on the adequacy of gravity models to describe human mobility in an epidemiological context).

Some of the parameters of model (1) - namely those related to human demography $(\mu)$ and the physiological response to the disease $(\alpha, \gamma, K)$-are assumed to be constant over the spatial and temporal scales considered in this study. The size of local human communities $\left(H_{i}\right)$ is assumed to possibly vary in space but not in time, at least over timescales of epidemiological interest. Some other parameters-namely the exposure and contamination rates $\beta_{i}(t)$ and $p_{i}(t)$, the pathogen mortality rate $\mu_{B i}(t)$, the hydrological movement rate $l_{i}(t)$ and bias $b_{i}(t)$, the fraction of traveling people $m_{i}(t)$ and the average movement distance $D_{i}(t)$, and the volume of the local water reservoir $W_{i}(t)$ - can vary in time and/or space. We restrict our analysis to periodical fluctuations linked to seasonal environmental drivers. The period of the environmental fluctuations is assumed to be 1 year without loss of generality. While sinusoidal oscillations may provide a simplistic representation of the natural frequencies of the relevant environmental drivers, they nevertheless capture the essence of the issue addressed-whether a time-varying framework affects macroscopically the conditions for waterborne pathogen invasion and the resulting patterns of infection.

\section{Derivation of pathogen invasion conditions}

Pathogens can invade the system if and only if the diseasefree equilibrium $\mathbf{X}_{\mathbf{0}}$, i.e., a state of model (1) where $S_{i}=$ $H_{i}, I_{i}=0$ and $B_{i}=0$ for all $i=1, \ldots, n$, is unstable under the assumption of periodic parameter fluctuations. To analyze the stability of $\mathbf{X}_{\mathbf{0}}$, we consider the linearized system

$$
\begin{aligned}
\frac{d \Delta \mathbf{S}}{d t} & =-\mu \Delta \mathbf{S}-\left[\mathbf{U}_{\mathbf{n}}-\mathbf{m}(t)\right] \mathbf{H} \boldsymbol{\beta}(t) \mathbf{B}^{*}-\mathbf{m}(t) \mathbf{H Q}(t) \boldsymbol{\beta}(t) \mathbf{B}^{*} \\
\frac{d \mathbf{I}}{d t} & =\left[\mathbf{U}_{\mathbf{n}}-\mathbf{m}(t)\right] \mathbf{H} \boldsymbol{\beta}(t) \mathbf{B}^{*}+\mathbf{m}(t) \mathbf{H} \mathbf{Q}(t) \boldsymbol{\beta} \mathbf{B}^{*}-(\gamma+\mu+\alpha) \mathbf{I}
\end{aligned}
$$

$$
\begin{aligned}
\frac{d \mathbf{B}^{*}}{d t} & =-\left[\boldsymbol{\mu}_{\mathbf{B}}(t)+\mathbf{l}(t)\right] \mathbf{B}^{*}+\mathbf{W}^{-1}(t) \mathbf{P}^{T}(t) \mathbf{W}(t) \mathbf{l}(t) \mathbf{B}^{*}+ \\
& +\frac{1}{K} \mathbf{p}(t) \mathbf{W}^{-1}(t)\left[\mathbf{U}_{\mathbf{n}}-\mathbf{m}(t)+\mathbf{Q}^{T}(t) \mathbf{m}(t)\right] \mathbf{I},
\end{aligned}
$$

where the superscript $T$ indicates matrix transposition; $\mathbf{U}_{\mathbf{n}}$ is the identity matrix of dimension $n ; \Delta \mathbf{S}=$ $\left[S_{1}-H_{1}, \cdots, S_{n}-H_{n}\right]^{T} ; \mathbf{I}=\left[I_{1}, \cdots, I_{n}\right]^{T} ; \mathbf{B}^{*}=$ $\left[B_{1} / K, \cdots, B_{n} / K\right]^{T}$; and $\mathbf{H}, \boldsymbol{\beta}(t), \mathbf{p}(t), \boldsymbol{\mu}_{\mathbf{B}}(t), \mathbf{l}(t), \mathbf{m}(t)$, $\mathbf{W}(t)$ are diagonal matrices whose nonzero elements are made up by the parameters $H_{i}, \beta_{i}(t), p_{i}(t), \mu_{B i}(t), l_{i}(t)$, $m_{i}(t), W_{i}(t)$, with $i=1,2, \cdots, n$, respectively.

Because of the block-triangular structure of the Jacobian of system (2), in which all but the first $n$ elements of the first $n$ columns are identically equal to 0 , we can evaluate the stability of the disease-free equilibrium $\mathbf{X}_{\mathbf{0}}$ applying Floquet theory to the subsystem $\mathcal{S}$ that includes the linearized equations for the "infected" compartments of the model (see also Bacaër 2007; Wang and Zhao 2008), namely infective abundances $I_{i}$ and pathogen concentrations $B_{i}^{*}$. Specifically, $\mathbf{X}_{\mathbf{0}}$ is unstable (thus allowing pathogen invasion) if and only if the maximum Floquet exponent $\xi_{\max }$ is larger than zero (e.g., Bittanti and Colaneri 2009). The procedure used to compute the Floquet exponents is detailed in Appendix S1 (section S1.1, Online Resources).

Note that this derivation of the invasion threshold is similar to one proposed by Bacaër (2007) (see Section 3.4 therein). Conversely, we do not set our analysis in the nextgeneration matrix framework, i.e., we do not decompose the matrix of the linearized system into transmission and transition parts, as done, e.g., in Wang and Zhao (2008). This decomposition might in fact be nontrivial for waterborne diseases involving free-living pathogen stages, especially if the pathogen population can self-sustain (Bani-Yaghoub et al. 2012). Also, it has been shown that linear stability 
analysis and the next-generation matrix approach (of which Floquet theory and the work by Wang and Zhao 2008 provide extensions) can lead to the same threshold results for spatially explicit models of waterborne disease dynamics (Gatto et al. 2013).

\section{Analysis of the invasion conditions: local model}

Floquet exponents vary as a function of the model parameters - and so obviously do pathogen invasion conditions. We illustrate the analysis of these conditions starting from the simple case of model (1) with no spatial structure and with periodic fluctuations of one of the model parameters expressed as

$\theta(t)=\theta_{0}[1 \pm \epsilon \sin (2 \pi t)]$.

Similar spatially implicit models for waterborne disease dynamics have been analyzed in Righetto et al. (2012) and Zhou and Cui (2013) with particular focus on longterm dynamics and the global stability of periodic solutions, respectively. To obtain a spatially implicit version of model (1), it is sufficient to consider the dynamics in the $i$-th node and to set $l_{i}(t)=0$ and $m_{i}(t)=0$ for all $t$, i.e., to disregard pathogen transport and human mobility. The stability of the disease-free equilibrium $\mathbf{X}_{\mathbf{0}}=\left[\begin{array}{lll}H & 0 & 0\end{array}\right]^{T}$ is thus determined by the maximum Floquet exponent of the following linearized subsystem $\mathcal{S}^{\prime}$ accounting for the local dynamics of infectives and pathogen concentrations:

$$
\begin{aligned}
\frac{d I}{d t} & =\beta(t) H B^{*}-(\gamma+\mu+\alpha) I \\
\frac{d B^{*}}{d t} & =-\mu_{B}(t) B^{*}+\frac{p(t)}{K W(t)} I
\end{aligned}
$$

where $B^{*}=B / K$. A step-by-step guide for the computation of Floquet exponents for the local model is given in Appendix S1 (section S1.2), together with a basic implementation of the numerical algorithm in MATLAB ${ }^{\circledR}$ language (section S1.3).

Local pathogen invasion conditions in periodic environments are shown in Fig. 2, where the invasibility curves (identified by the condition $\xi_{\max }=0$ ) are reported for different assumptions regarding the time-varying parameter, taken in the form of Eq. (3) (note that the sign is immaterial for invasion dynamics). Depending on the parameter that is assumed to vary over time, in fact, periodic fluctuations can make pathogen invasion either less $(\beta, p)$ or more likely $\left(\mu_{B}, W\right)$, i.e., occurring for either larger or smaller average values of the time-varying parameter than in the time-constant case $\left(\beta(t)=\beta_{0}, \mu_{B}(t)=\mu_{B 0}, p(t)=p_{0}\right.$, $\left.W(t)=W_{0}\right)$. Therefore, simplistic conclusions like "seasonality favors [plays against] pathogen invasion" cannot be drawn because the effect of seasonality will depend on which of the drivers (of either biotic or abiotic nature) fluctuate seasonally. Figure 2 also shows that the effects on pathogen invasion of time-varying exposure and contamination rates are qualitatively indistinguishable from each other (panels a and $\mathrm{b}$, where the analytical approximation for the invasion threshold proposed in Section 3.3 of Bacaër 2007 is also reported).

In a time-constant and spatially implicit environment, the condition for pathogen invasion is

$R_{0}=\frac{\beta_{0} p_{0} H}{\mu_{B 0}(\gamma+\mu+\alpha) K W_{0}}>1$,

i.e., pathogen invasion is possible if and only if the basic reproduction number $R_{0}$ is larger than one (see, e.g., Codeço 2001). In a periodically fluctuating environment, one can still calculate an "approximate" $\widetilde{R}_{0}$ using the values of the model parameters averaged over time, but the condition $\widetilde{R}_{0}>1$ will no longer correspond to pathogen invasion because the maximum Floquet exponent must be evaluated instead. However, from now on, we will still term "basic reproduction number" this approximate $\widetilde{R}_{0}$ because it allows a straightforward comparison of the invasion thresholds obtained in a periodic environment (and, in the sequel, in a spatially explicit setting) with those relevant to the simple case of constant parameters (and no spatial structure). The assessment of the invasion thresholds can be usefully complemented by the analysis of the initial dynamics of infection conducted via simulation. For the numerical experiments, we use the initial condition $S(0)=H-I(0)$, $I(0)=10^{-3} H$, and $B^{*}(0)=0$ and introduce environmental fluctuations that increase infection risk at the beginning of the simulation timespan (i.e., initially increasing [decreasing] exposure [pathogen mortality/water volumes]). These experiments allow us to assess the severity of a waterborne disease epidemic. Specifically, the size of an outbreak can be measured in terms of the attack ratio $Y$, here defined as the annual incidence normalized by total population, i.e.,

$Y=\frac{1}{H} \int_{0}^{1} \beta(t) \frac{B^{*}(t)}{K+B^{*}(t)} S(t) d t$.

Figure 3 reports the invasibility curves of Fig. 2 redrawn as a function of $\widetilde{R}_{0}$. In the case of temporally varying pathogen mortality rate $\mu_{B}$ or water volume $W$ [exposure $\beta$ or contamination rate $p$ ], the value of $\widetilde{R}_{0}$ for which $\xi_{\max }=0$ is smaller [larger] than one for $\epsilon>0$. Therefore, sub/super-threshold pathogen invasions (i.e., occurring for $\widetilde{R}_{0}$ smaller or larger than one, respectively; see, e.g., van den Driessche and Watmough 2002; Gatto et al. 2012, 2013) 
Fig. 2 Effects of parameter fluctuations on the pathogen invasion threshold. a Periodic fluctuations (Eq. (3)) of the exposure rate $\beta$. The solid black line represents the invasibility curve, along which $\xi_{\max }=0$. Pathogens can invade for parameter combinations lying in the gray-shaded area. $\mathbf{b}-\mathbf{d}$ As in panel a, respectively for periodic fluctuations of contamination rate $p$ (panel b), pathogen mortality rate $\mu_{B}$ (panel c), and water reservoir $W$ (panel d), whose average volume is assumed to be proportional to population abundance $H$ $(\bar{W}=c H)$. The dotted curves in panels $\mathbf{a}$ and $\mathbf{b}$ have been computed with the analytical approximation proposed by Bacaër (2007). Baseline parameter values: $\beta_{0}=1$, $\mu=5 \cdot 10^{-5}, \alpha=5 \cdot 10^{-4}$, $\gamma=\mu_{B 0}=0.2$,

$p /(K c)=0.04, H=1$. All rates are chosen to reproduce the basic timescales of cholera dynamics (e.g., Mari et al. 2012a) and are expressed as days $^{-1}$ for the sake of clarity
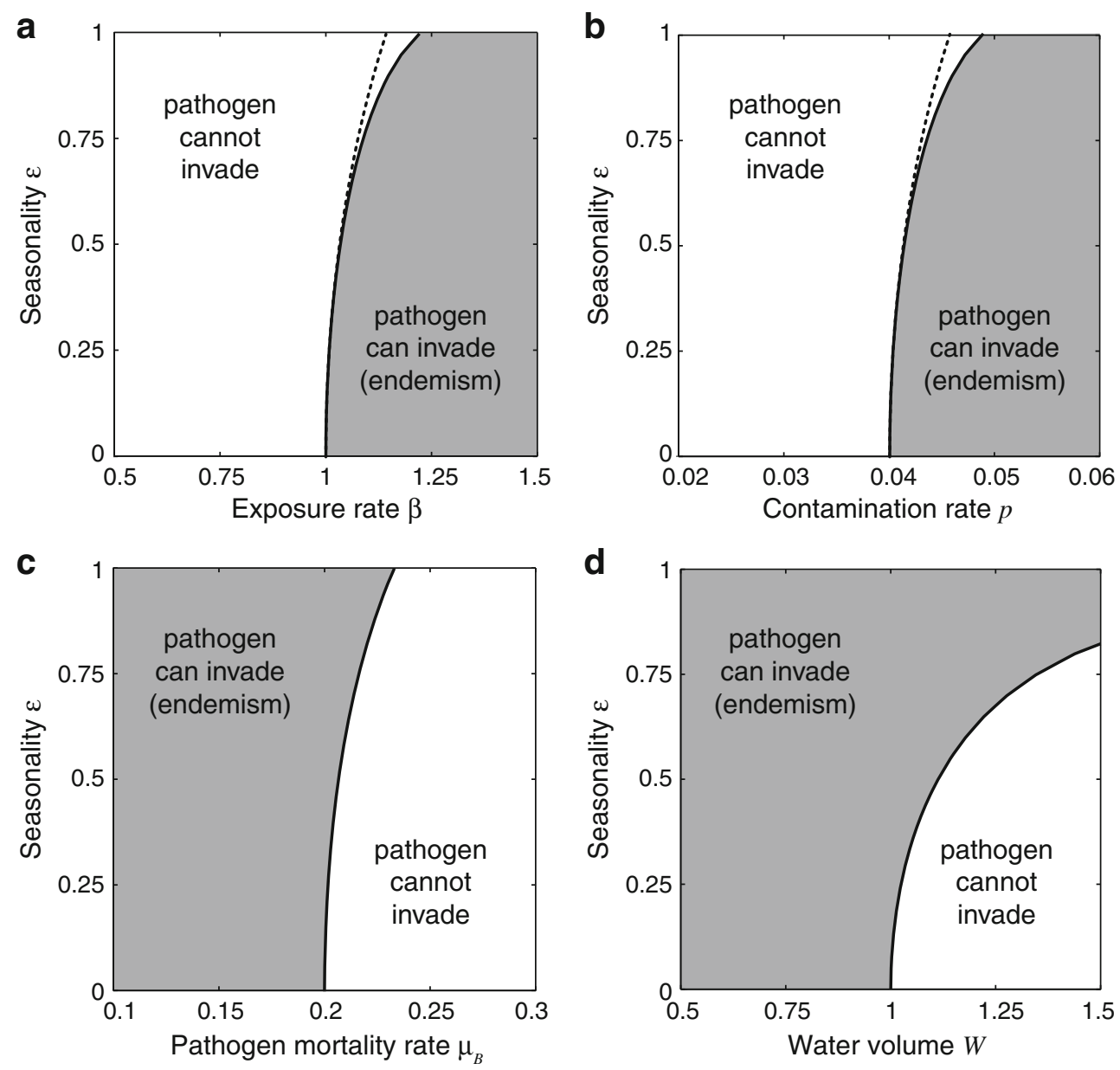

are possible, provided that temporal variability is accounted for. Figure 3 also shows that in some cases, parameter fluctuations can indeed trigger epidemics whose size is considerably large even if $\xi_{\max }<0$ (see again Bacaër and Gomes 2009). We can term these cases "outbreaks without endemism" (OwE). They are identified in Fig. 3 by $\xi_{\max }<0$ and, conventionally, $Y \geq 0.5$. This might seem very high a number, but it must be noticed that our definition of attack ratio also accounts for asymptomatic cases, which can be a large share of the total number of infections in several waterborne diseases such as cholera (about $75 \%$ of all cases according to World Health Organization 2010) and amoebiasis (about $80 \%$ for infections caused by Entamoeba histolytica; see Guerrant 1986). Cases of OwE are particularly interesting because they signal conditions for which the analysis of invasion thresholds alone would have failed to identify epidemic waves triggered by transient dynamics, possibly characterized by very large attack ratios. Conversely, there are parameter combinations for which $\xi_{\max }>0$, yet the size of the ensuing epidemic is rather small. These cases are termed "endemisms without outbreak" (EwO) and are identified by $\xi_{\max }>0$ and $Y<0.5$ in Fig. 3.

\section{Analysis of the invasion conditions: network model}

Some results for very simple network topologies are given in Appendix S1 (section S1.4 and Fig. S1). Here, we proceed to the analysis of pathogen invasion conditions for the full network model applied to a realistic river network using the general method illustrated in "Derivation of pathogen invasion conditions". Specifically, we consider two different cases: (i) space-time fluctuations of one single parameter and (ii) coupled fluctuations of two parameters over time. As for case (i), we assume that the space/time-varying parameter (generically termed $\theta_{i}(t)$ ) is described by

$\theta_{i}(t)=\bar{\theta}_{i}\left[1 \pm \epsilon \sin \left(2 \pi t+\phi \pi \delta_{i}\right)\right]$,

where $\bar{\theta}_{i}$ is the average value of $\theta_{i}(t), 0 \leq \epsilon<1$ and $\phi \geq 0$ quantify amplitude and lag of space-time oscillations, and $\delta_{i}$ is a parameter suitably accounting for the lag dependence on the position of node $i$ within the spatial domain. As for case (ii), we assume that the two parameters $\theta_{i}^{\prime}(t)$ and $\theta_{i}^{\prime \prime}(t)$ follow

$$
\begin{aligned}
\theta_{i}^{\prime}(t) & =\bar{\theta}_{i}^{\prime}\left[1+\epsilon^{\prime} \sin (2 \pi t)\right] \\
\theta_{i}^{\prime \prime}(t) & =\bar{\theta}_{i}^{\prime \prime}\left[1+\epsilon^{\prime \prime} \sin \left(2 \pi t+\phi^{\prime \prime} \pi\right)\right]
\end{aligned}
$$



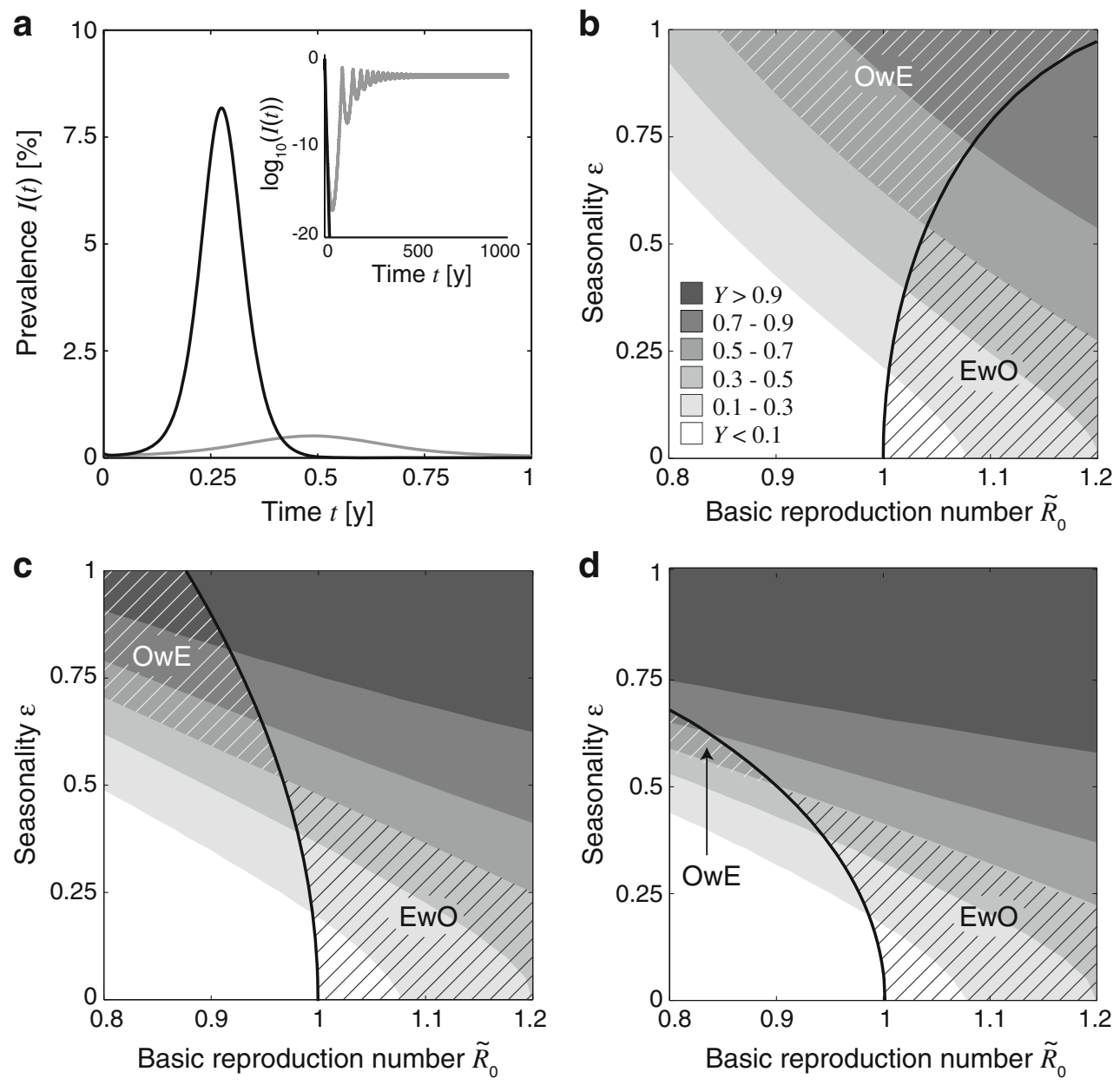

Fig. 3 Simulation of epidemic dynamics and analysis of pathogen invasion conditions for the time-varying local model. a Numerical simulations obtained with a periodic exposure rate $\beta$ : the black curve is an example of outbreak without endemism $\left(\mathrm{OwE}, \widetilde{R}_{0}=1.1, \epsilon=0.9\right.$, corresponding to $\xi_{\max }<0$ ), while the gray curve is an example of endemism without outbreak (EwO, $\widetilde{R}_{0}=1.1, \epsilon=0.1$, corresponding to $\xi_{\max }>0$ ). Inset Long-term dynamics, showing pathogen extinction (black) or persistence (gray). b Effect of periodic fluctuations (Eq. (3)) of exposure rate on the invasion threshold (solid black) and the yearly

where $\bar{\theta}_{i}^{\prime}$ and $\bar{\theta}_{i}^{\prime \prime}$ are the average values of $\theta_{i}^{\prime}(t)$ and $\theta_{i}^{\prime \prime}(t)$, respectively, $0 \leq \epsilon^{\prime}<1$ and $0 \leq \epsilon^{\prime \prime}<1$ quantify the amplitude of seasonal fluctuations, and $0 \leq \phi^{\prime \prime} \leq 1$ is the lag between $\theta_{i}^{\prime}(t)$ and $\theta_{i}^{\prime \prime}(t)$. In the reminder of the paper, the average value(s) of the time-varying parameter(s) will be assumed to be spatially homogeneous (i.e., $\bar{\theta}_{i}=\theta_{0}, \bar{\theta}_{i}^{\prime}=\theta_{0}^{\prime}$, $\left.\bar{\theta}_{i}^{\prime \prime}=\theta_{0}^{\prime \prime}\right)$, except for the average volumes of the local water reservoirs, that will be assumed to be proportional to local population abundances $\left(\bar{W}_{i}=c H_{i}\right.$, Bertuzzo et al. 2010).

\section{Single-parameter fluctuations}

Figure 4 reports the invasibility curves computed for different parameter values and different assumptions regarding one single time-varying parameter, here taken in the form

attack ratio $Y$ of the epidemic (gray shadings, see legend); pathogens can invade for parameter sets on the right of the invasibility curve, along which $\xi_{\max }=0$. $\mathbf{c}$ As in panel a, for periodic fluctuations of pathogen mortality rate $\mu_{B}$. d As in panel a, for periodic fluctuations of water reservoir $W$. Periodic fluctuations are chosen so as to increase infection risk at the beginning of the simulation period (panels $\mathbf{a}$ and $\mathbf{d}$ : $\theta(t)=\bar{\theta}[1+\epsilon \sin (2 \pi t)] ;$ panels b and $\mathbf{c}: \theta(t)=\bar{\theta}[1-\epsilon \sin (2 \pi t)])$. Other parameters as in Fig. 2

of Eq. (4), in a network in which population distribution is assumed to be spatially homogeneous $\left(H_{i}=H\right.$ for all $\left.i\right)$. If $\epsilon=0$ (constant parameter values), pathogen invasion is possible if and only if $\widetilde{R}_{0}>\lambda_{0}$, with $\lambda_{0}$ being the value of $\widetilde{R}_{0}$ for which the dominant eigenvalue $\Lambda_{0}$ of the generalized reproduction matrix of size $2 n$ introduced by Gatto et al. $(2012,2013)$ is equal to zero. This matrix represents the time-invariant counterpart of matrix $\mathbf{J}_{\mathbf{0}}(t)$ defined above, therefore it accounts not only for epidemiological dynamics but also for spatial connectivity. Note that, for this parameter setting, $\lambda_{0}$ is larger than 1 , which implies that pathogen transport and human mobility impose a stricter invasion condition than the one derived in the simple case of no spatial connectivity and no temporal variability $\left(R_{0}>1\right)$. This result indicates that the spatiotemporal dynamics of 


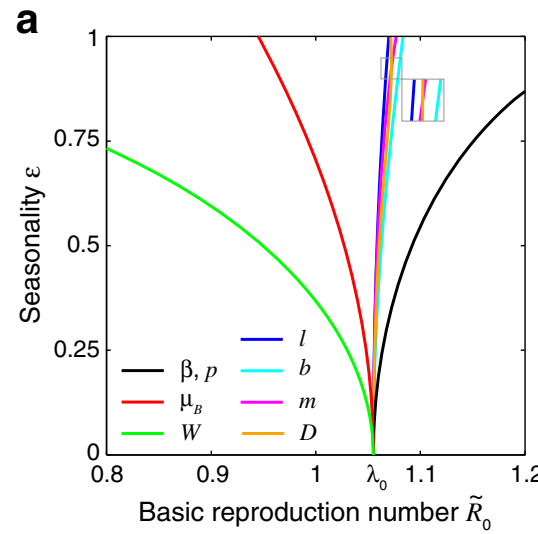

b

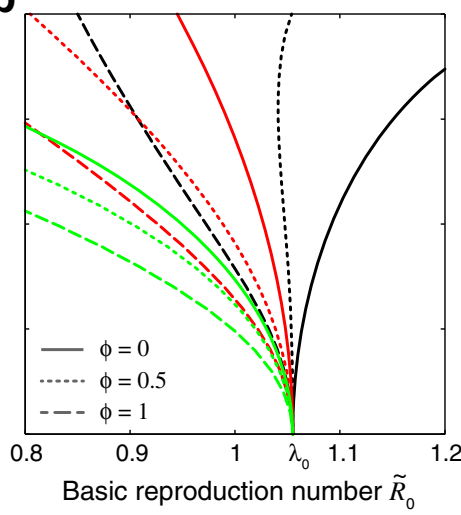

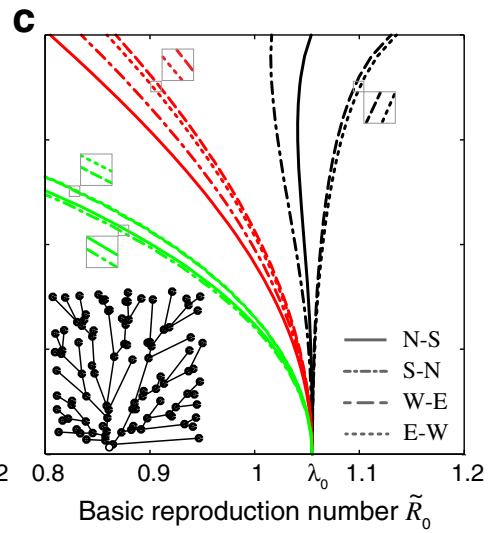

Fig. 4 Analysis of pathogen invasion conditions for spatiotemporal periodic fluctuations of the parameters (Eq. (4)) in the full network model applied to a realistic river network. Pathogens can invade for parameter combinations on the right of the invasibility curves (along which $\left.\xi_{\max }=0\right)$. a Effect of synchronous $(\phi=0)$ fluctuations of different parameters (color coded). b Effect of different lags $\phi$ for $\delta_{i}$ following a N-S gradient (see text for details). c Effect of different gradient directions for the spatiotemporal fluctuations of the model

the epidemiological process in our dendritic network share some basic properties with contact processes (Harris 1974) or spatially explicit metapopulations (Casagrandi and Gatto 2006; Mari et al. 2014).

More importantly, Fig. 4 shows that, if $\epsilon>0$, fluctuations of the environmental conditions, subsumed by fluctuations of the model parameters, can remarkably influence pathogen invasion in a spatially explicit setting. Pathogen invasion can in fact be either favored (i.e., expected to occur for values of $\widetilde{R}_{0}$ lower than $\lambda_{0}$ ) or made more difficult, depending on the parameter assumed to vary in time (panel a). Specifically, periodic fluctuations of the parameters that appear at the denominator of $\widetilde{R}_{0}$ (namely pathogen mortality and water volume) favor pathogen invasion, while fluctuations of the other parameters considered here play against it. Remarkably, for sufficiently large values of $\epsilon$, fluctuations of pathogen mortality or water volume can induce pathogen invasion not only for $\widetilde{R}_{0}<\lambda_{0}$ but also for $\widetilde{R}_{0}<1$, i.e., for values of the parameters that would not allow it in a local model (a case of subthreshold epidemic, see above).

These results hold true not only for a spatially homogeneous population distribution but also for more realistic spatial arrangements. For instance, the size of each local community can be set so as to follow the so-called Zipf's law (Zipf 1949), according to which the probability density function of local population sizes decays proportionally to $H^{-2}$. The remarkable feature of Zipf's distribution is that it is suggested to accurately describe the sizes of cities of any country in a purportedly universal fashion regardless of geographical, environmental, social, or economic conditions, once properly corrected for the finite size effect induced by total population (Newman 2005; see also parameters $(\phi=0.5)$. In panels $\mathbf{b}$ and $\mathbf{c}$, colors are the same as in panel a. Population is assumed to be homogeneously distributed $\left(H_{i}=H\right)$ over the river network (inset of panel c; the network has been obtained by extracting from an OCN the 100 nodes with largest cumulative drainage area), and the average volume of local water reservoirs is proportional to local population size $\left(\bar{W}_{i}=c H_{i}\right)$. Parameter values as in Fig. 3. Other parameters: $l_{0}=0.5\left[\mathrm{day}^{-1}\right], b_{0}=0.5, m_{0}=0.5$, $D_{0}=0.1$ (fraction of domain length), $H=1$

Bertuzzo et al. 2010; Gatto et al. 2012; Mari et al. 2012b; Gatto et al. 2013 for relevant epidemiological applications). For the sake of simplicity, here, we do not introduce any (likely yet nontrivial) correlation of population size and position in the river network. Also, to ease comparison with the spatially homogeneous case, the average reservoir volumes are assumed to be proportional to the size of the local communities $\left(\bar{W}_{i}=c H_{i}\right)$, and the total population abundance is set to be the same in the two different spatial arrangements (i.e., $\sum_{i=1}^{n} H_{i}=n H$ ). Figure S2 shows that a heterogeneous population distribution is expected to favor pathogen invasion, as already found in a time-invariant setting elsewhere (Gatto et al. 2012, 2013).

While the spatially explicit case retains most of the properties outlined for the implicit case, new additional features of disease dynamics clearly emerge in the analysis of the network model. In particular, a space-dependent temporal delay in environmental fluctuations $(\phi>0$, Eq. 4) can determine a heterogeneous spatial distribution for the timevarying parameter in large river basins. This heterogeneity may have important implications for pathogen invasion, as shown in Fig. 4b, in which $0 \leq \delta_{i} \leq 1$ (Eq. (4)) is supposed to increase from the bottom (i.e., where the network outlet lies, see inset of panel c) to the top of the spatial domain (north-south, N-S, direction), so that $\theta_{i}(t)$ is characterized by early peaks moving northward from the outlet. Increasing spatiotemporal asynchrony of the seasonal fluctuations (represented by increasing values of $\phi$ ) favor pathogen invasion, with subthreshold epidemics being possible for sufficiently large values of $\epsilon$ and $\phi$. Different spatial patterns of parameter fluctuations (represented by different choices of geographic gradients $\delta_{i}$ ) may also matter. Figure 
$4 \mathrm{c}$ shows in fact that invasion thresholds can be quite different for different directions of the spatial perturbations. A clear difference emerges for latitudinal vs. longitudinal patterns, with the former leading to lower invasion thresholds for all tested time-varying parameters. As for latitudinal patterns, it does not seem possible to infer a general rule, with $\mathrm{N}-\mathrm{S}$ variations leading to higher invasion thresholds than those in the $\mathrm{S}-\mathrm{N}$ directions for some parameters $(\beta, p)$, to lower thresholds for others $\left(\mu_{B}\right)$-and being almost indistinguishable for yet others $(W)$. As for longitudinal patterns, which are "orthogonal" to the main direction of the water flow, the invasion thresholds obtained for west-east (W-E) vs. E-W perturbations are remarkably similar. This finding is not surprising given the almost-symmetric structure of the OCN used in the numerical experiments. Quantitative details aside, most of these findings are valid for different choices of the baseline epidemiological parameters. As an example, Fig. S3 reports the results for a different parameter setting, in which $\lambda_{0}<1$.

The analysis of the invasion conditions can be usefully complemented by numerical simulations of the network model (Fig. 5). Simulations have been initialized by assuming that a small fraction $(0.1 \%)$ of the population living in the outlet node of the river network is initially infected and that infection risk is increasing at the beginning of the model simulation. Epidemic size, here measured as

$$
\begin{aligned}
Y= & \frac{1}{\sum_{i=1}^{n} H_{i}} \sum_{i=1}^{n} \int_{0}^{1}\left\{\left[1-m_{i}(t)\right] \beta_{i}(t) \frac{B_{i}^{*}(t)}{K+B_{i}^{*}(t)}\right. \\
& \left.+m_{i}(t) \sum_{j=1}^{n} Q_{i j}(t) \beta_{j}(t) \frac{B_{j}^{*}(t)}{K+B_{j}^{*}(t)}\right\} S_{i}(t) d t,
\end{aligned}
$$

and pathogen invasion are not necessarily related to each other also in a spatially explicit setting. Rather, for the parameter combinations explored in Fig. 5, large epidemics are expected to develop even for low values of $\widetilde{R}_{0}$, corresponding in fact to $\xi_{\max }<0$, provided that the amplitude of seasonal fluctuations $\epsilon$ is sufficiently large. This phenomenon is more remarkable for fluctuations of $\beta$ and $W$, less for fluctuations of $\mu_{B}$. Qualitatively similar results are also found for a spatially heterogeneous population distribution following Zipf's law (Fig. S4). However, contrasting Fig. 5 to Fig. S4 shows that, all the other things being equal, a heterogeneous population is much more likely to be hit by a large epidemic than a homogeneously distributed one.

\section{Coupled-parameter fluctuations}

It is also interesting to briefly analyze the case in which two parameters fluctuate periodically over time (according to Eq. (5) above) as a response to different environmental drivers. For instance, rainfall and temperature patterns may be characterized by different amplitude and timing and can produce different effects on the model parameters (with rainfall influencing, e.g., water volumes and/or exposure rates and temperature affecting pathogen mortality rates), thus affecting in turn eco-epidemiological dynamics. Phase lag can introduce complexities in the long-term patterns of seasonally forced ecosystems not only at the local scale (Rinaldi and Muratori 1993) but also in real, spatially explicit settings. As an example, cholera incidence in some regions of the Indian subcontinent exhibits two annual peaks, although each of the main environmental drivers linked to the disease in the area (river discharge, temperature, zooplankton abundance) peak only once per year (Akanda et al. 2009). Results from a modeling study

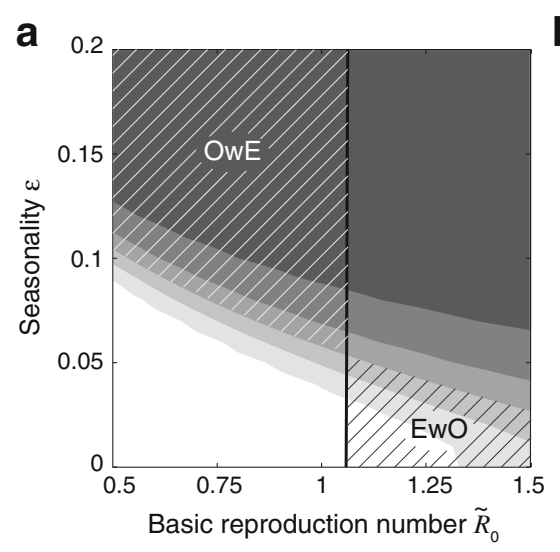

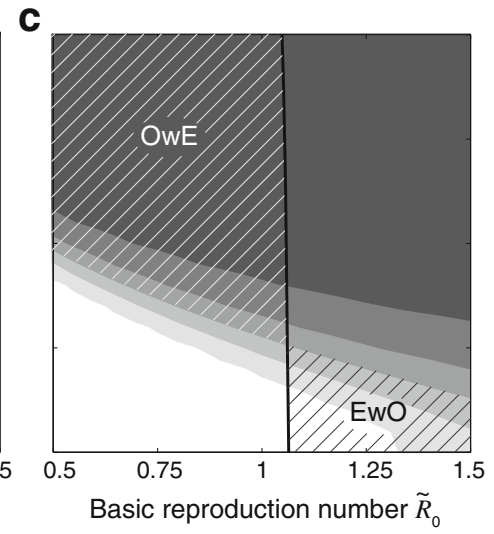

Fig. 5 Simulation of epidemic dynamics for the network model with time-varying parameters. a Effect of periodic fluctuations (Eq. (4) with $\phi=0$ ) of the exposure rate $\beta$ on the yearly attack ratio $Y$ of the epidemic (gray shadings, see legend). b As in panel a, for periodic fluctuations of the pathogen mortality rate $\mu_{B}$. $\mathbf{c}$ As in panel $\mathbf{a}$, for periodic fluctuations of the water reservoir $W$. Periodic fluctuations are chosen so as to increase infection risk at the beginning of the simulation period (panel a: $\theta_{i}(t)=\bar{\theta}_{i}[1+\epsilon \sin (2 \pi t)]$; panels $\mathbf{b}$ and $\mathbf{c}$ : $\left.\theta_{i}(t)=\bar{\theta}_{i}[1-\epsilon \sin (2 \pi t)]\right)$. Invasibility curves (solid black lines) correspond to those already shown in Fig. 4 a (note that axes have different scales). Other details and parameter values as in Fig. $4 \mathrm{a}$ 
(Bertuzzo et al. 2012) indicate that the interaction among these drivers is fundamental to explain emergence and localization of double-peak patterns of cholera incidence.

Figure 6 reports the conditions for pathogen invasion to occur $\left(\xi_{\max }>0\right)$ for periodic fluctuations of either exposure or pathogen mortality rates coupled to periodic fluctuations of the local water reservoirs in the network model with homogeneous population distribution. Our analysis shows that, for a given amplitude of the seasonal fluctuations of the water reservoir volumes, a marked synchronous seasonality of the exposure rate $(\beta)$ contrasts pathogen invasion (panel a). This result can be explained by noting that in this setting, exposure is high [low] when pathogen dilution is also high [low], so that a compensation might occur between these two components of disease transmission. Conversely, ample fluctuations of pathogen mortality $\left(\mu_{B}\right)$ favors pathogen invasion for a given amplitude of the fluctuations of the water reservoir (panel b). In this case, the synchrony between low water volumes and low pathogen mortality rates most likely has a disproportionate effect toward pathogen invasion. Seasonal environmental drivers can thus play either synergistically or antagonistically to set the invasion threshold because of specific eco-epidemiological interactions between model parameters. Differences in the timing of the fluctuations can be important as well. As an example, Fig. 6c shows that increasing the delay between the fluctuations of the local water reservoirs $(W)$ and the exposure rates makes pathogen invasion more likely. In this case, in fact, asynchrony makes the aforementioned compensation between exposure and pathogen concentration less effective. Note that there also exist cases in which timing does only marginally influence pathogen invasion, as documented in Fig. $6 \mathrm{~d}$.

\section{Spatial patterns of epidemic spread}

Defining the conditions for pathogen invasion represents an important step toward a better understanding of waterborne disease outbreaks. However, the identification and possible prediction of the spatial patterns of initial epidemic spread becomes crucial in operational terms, e.g., for early allocation of health care staff and supplies. In the simpler case of time-constant parameter values, the spatial signature of an epidemic outbreak has been linked (Gatto et al. 2012, 2013) to the dominant eigenvector of a generalized reproduction
Fig. 6 Analysis of pathogen invasion conditions for coupled periodic fluctuations of pairs of model parameters (Eq. (5)) in the network model. Pathogens can invade $\left(\xi_{\max }>0\right)$ for parameter combinations lying on the right of the invasibility curves, computed for different values of $\widetilde{R}_{0}$ (legend). a Synchronous fluctuations of local water reservoir volumes $W$ (characterized by amplitude $\epsilon_{W}$ ) and exposure rates $\beta$ (with amplitude $\epsilon_{\beta}$ and phase $\phi_{\beta}=0$ ). b As in $\mathbf{a}$, for synchronous fluctuations of water reservoirs and pathogen mortality $\mu_{B}$ (characterized by amplitude $\epsilon_{\mu_{B}}$ and phase $\phi_{\mu_{B}}=0$ ). c Asynchronous fluctuations of local water reservoirs and exposure rates (with amplitudes $\epsilon_{W}=\epsilon_{\beta}$ and phase $\phi_{\beta}$ ). $\mathbf{d}$ As in c, for asynchronous fluctuations of water reservoirs and pathogen mortality (with amplitudes $\epsilon_{W}=\epsilon_{\mu_{B}}$ and phase $\phi_{\mu_{B}}$ ). Other parameters as in Fig. 4
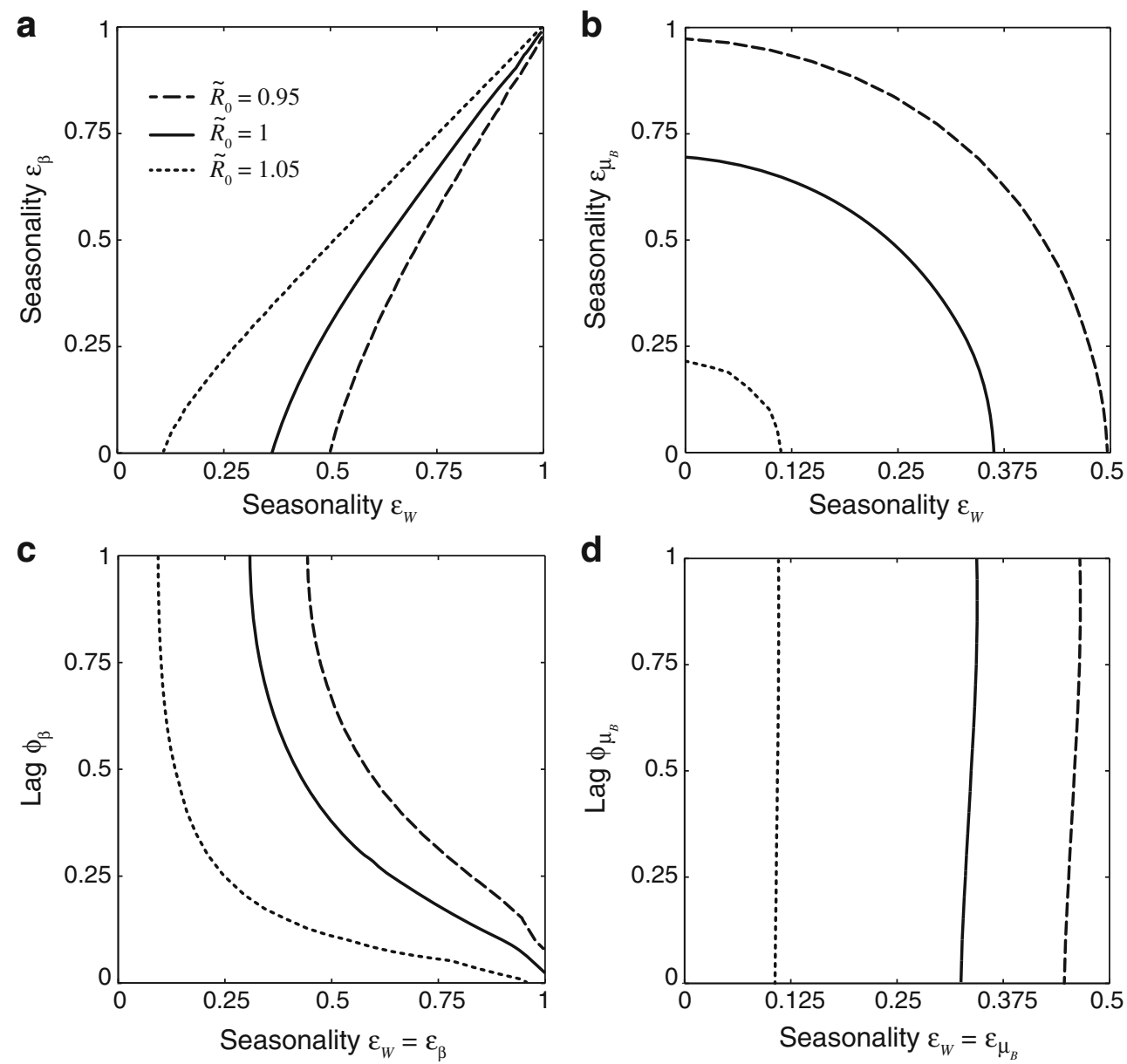
matrix accounting for epidemiological processes and the relevant pathogen relocation mechanisms. In fact, when the disease-free equilibrium is unstable, the dominant eigenvector of the generalized reproduction matrix pinpoints the direction in the state space along which the system orbit, after a transient due to initial conditions, will diverge from the equilibrium. By continuity with the time-constant case (see Wang and Zhao 2008), Floquet theory suggests that in the presence of time-varying parameters, the spatial patterns of pathogen invasion and epidemic outbreak will be given respectively by the infectives' and pathogens' components of the dominant eigenvector $\Xi$ of the monodromy matrix $\mathbf{F}$ (1) defined above. Note that, in analogy with the timeconstant case, transient dynamics can (at least partially) cloud the predictions of Floquet theory, especially in case of fast-developing epidemics that emerge soon after pathogen introduction.
The predictions of Floquet theory applied to the spatial patterns of epidemic spread can be easily verified by performing numerical simulations of model (1) and contrasting the infectives' components of $\Xi$ to the spatial distribution of disease prevalence at epidemic emergence, here defined as the point in time in which the sign of $d \sum_{i}^{n} I_{i} / d t$ switches from negative to positive in response to the periodic oscillation of the time-varying parameter (e.g., the volume of local water reservoirs in Fig. 7, see panels a and b). The comparison between eigenvector components and simulation results can be performed not only for a homogeneous population distribution (panel c) but also for other, more complex spatial patterns, even in case of outbreaks without endemism. As an example, panel $\mathrm{d}$ is obtained under the hypothesis that the size of local human communities follows Zipf's law. Other patterns of population distribution are analyzed in Fig. S5, namely population size proportional to cumulative
Fig. 7 Spatial patterns of epidemic outbreak. a Fluctuations around the reference value of the local water reservoir volumes $\Delta W_{i}(t)$. b Temporal pattern of total disease prevalence (semilog scale); the filled dots represent epidemic emergence as defined in the text. c Population density map (left, homogeneous population), components of the dominant eigenvector of the monodromy matrix $\mathbf{F}(1)$ (middle, $\xi_{\max }=2.04$ ), and spatial distribution of disease prevalence at epidemic emergence as simulated by model (1) (right). d As in panel c, for population density following Zipf's law

$\left(\xi_{\max }=4.82\right)$. The quantities shown in panels $\mathbf{c}$ and $\mathbf{d}$ have been normalized (so that the maximum has value 1 ). Parameters and simulation details as in Fig. 4a, with $\widetilde{R}_{0}=1, \epsilon=0.5, \phi=0$
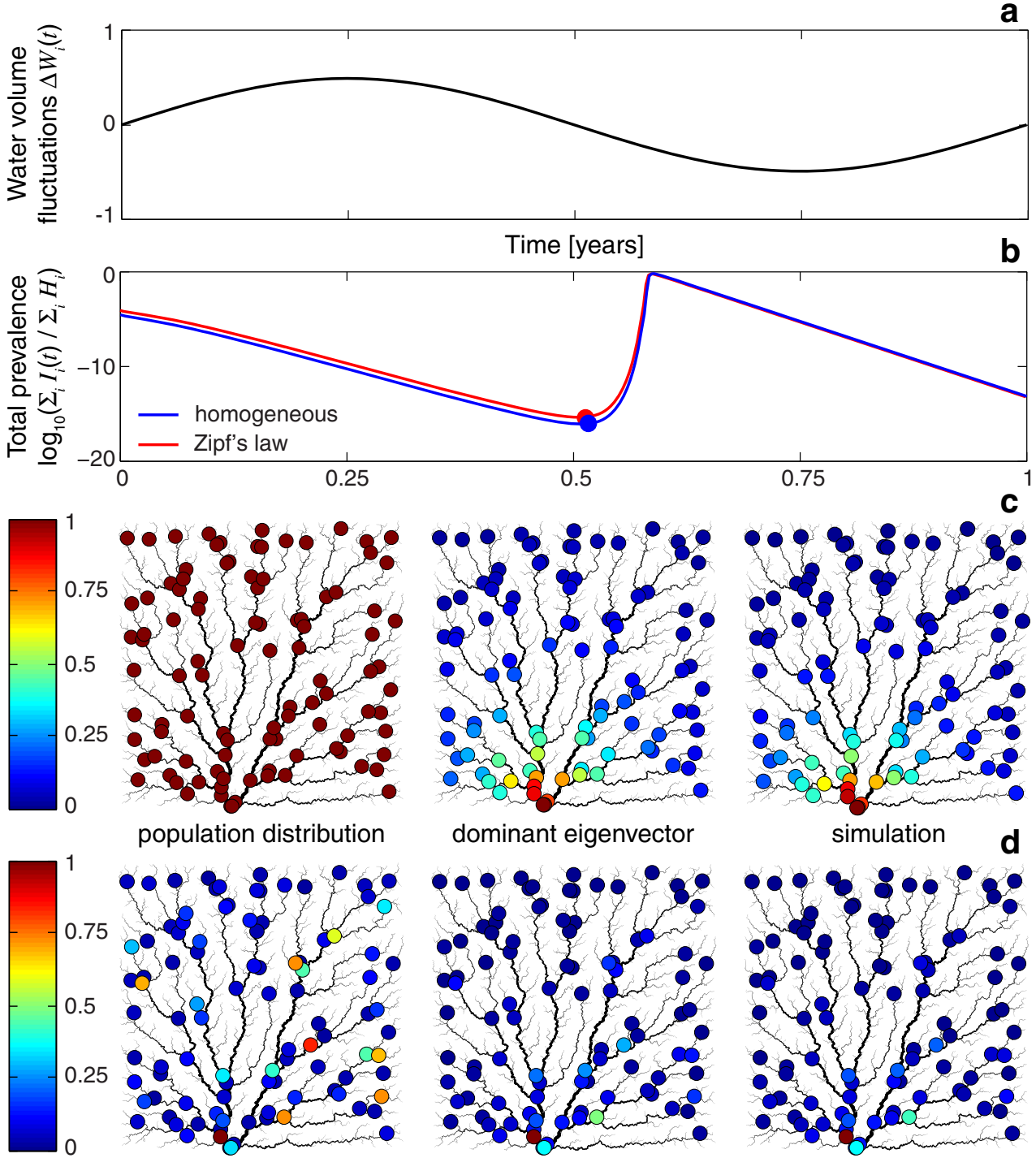

dominant eigenvector

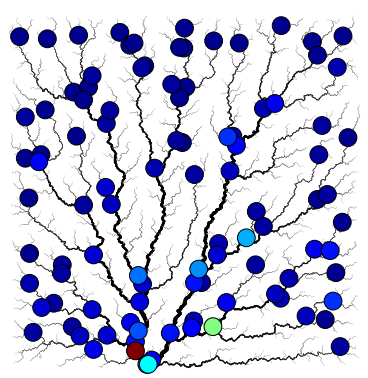

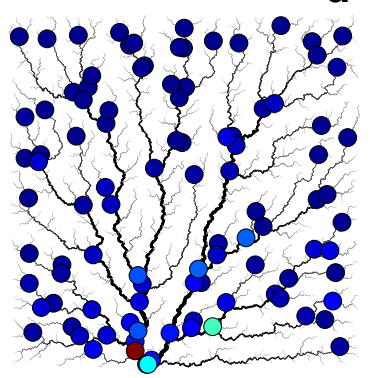


drainage area (panel c) or sampled from a random uniform distribution (panel d). Note that in the case of population proportional to drainage area, an epidemic outbreak may occur even if $\xi_{\max }<0$. However, the agreement between the components of $\Xi$ and simulation results is very satisfactory for every tested population distribution (coefficient of determination $R^{2} \geq 0.98$ for all cases). Similar correspondences are found also in the case of periodic fluctuations initially leading to an increase of infection risk (coefficient of determination $R^{2} \geq 0.95$ for all cases).

Note also that, despite differences in the underlying population distributions, the time series of total disease prevalence shown in Fig. 7b are very similar. Key to explaining this nontrivial result is the observation that epidemic emergence is triggered by increasing local pathogen concentrations resulting from a decrease in the volumes of the local water reservoirs. Once started, for $\epsilon \gg 0$ the course of the epidemic is very fast and "explosive." As the attack ratio approaches very high values $(>90 \%)$, the spatial distribution of human communities loses importance in comparison with less explosive epidemics.

\section{Discussion and conclusions}

In this work, we have analyzed invasion conditions for waterborne pathogens in spatially explicit and time-varying systems. Our approach is based on Floquet theory, an essential tool to study the stability of periodically forced dynamical systems (see, e.g., Bittanti and Colaneri 2009). Unlike linear stability analysis, Floquet theory has been somewhat neglected in ecology and epidemiology, especially with reference to realistic, spatially structured problems. However, seasonal fluctuations of environmental factors are crucial determinants of many ecological and epidemiological problems, notably including invasion conditions for competing species (Klausmeier 2008) or pathogenic organisms (Heesterbeek and Roberts 1995a, b; Bacaër 2007; Wang and Zhao 2008; Bacaër and Ait Dads 2012). In this framework, the quantity that actually controls pathogen invasion is the largest Floquet exponent $\xi_{\max }$ of the linearized system describing epidemiological dynamics. Specifically, the threshold condition is $\xi_{\max }>0$, which does not require that local $R_{0}$ values be larger than one. Subthreshold epidemics (van den Driessche and Watmough 2002) are thus possible. This invasion condition generalizes previous results obtained in a spatially explicit setting under the assumption of time-invariant dynamics (Gatto et al. 2012, 2013). Therefore, it is particularly suited to address the study of pathogen invasion whenever seasonal fluctuations of the relevant environmental drivers cannot be safely neglected. Numerical simulations are useful to complement the analysis of invasion conditions because in time-varying systems, long-term pathogen invasion is neither necessary nor sufficient for large epidemic outbreaks to occur.

We remark that accounting for environmental fluctuations can be crucially important, especially when dealing with pathogens in regions where the hydroclimatological drivers commonly linked to waterborne infections (e.g., temperature and rainfall) can be characterized by marked seasonal patterns. Our analysis shows that in those regions not only the amplitude but also the geographic signatures (e.g., the predominant direction) associated with the environmental fluctuations may contribute to defining the conditions under which waterborne pathogens can invade. Also, the likely coupling of environmental drivers, each with different seasonality, is expected to produce intriguing results, with implications for the analysis of real case studies, in which the identification of all the relevant seasonal drivers would thus be essential. Furthermore, seasonality can influence the spatial patterns of pathogen invasion. The importance of generalizing the concept of basic reproduction number not only to spatially explicit (Gatto et al. 2012, 2013) but also to time-varying systems (this study) is also demonstrated, in fact, by the ability of our approach to describe the geography of disease outbreaks, which is shown to be well characterized by the dominant eigenvector of the monodromy matrix. This result can be easily applied to other realistic landscapes described by networks of any given complexity (of which the present examples act as a proof of concept), ranging from a few to thousands of nodes.

The framework presented here could obviously be made more realistic in many respects. First, seasonal fluctuations are here represented as simple periodic signals, perhaps too crude an oversimplification of the erratic patterns often observed in time series of, e.g., temperature or rainfall intensity, which are known to influence pathogen demography, water availability, and exposure/contamination rates. Fourier expansion of more complex periodic signals is possible, at the expense of the clarity of analysis. However, overcoming this limitation would need a mathematical framework considerably more involved than Floquet theory, as it would require the (typically numerical) computation of the Lyapunov exponents associated with the disease-free equilibrium (e.g., Ferrière and Gatto 1995), or the use of suitable approximations to evaluate the long-term growth rate of the pathogen population (e.g., Tuljapurkar and Orzack 1980). We also remark that the techniques adopted in this paper cannot be reliably used to derive long-term epidemiological patterns. This task would in fact require the determination of the attractor(s) of the model and the analysis of their dynamical characteristics (e.g., via bifurcation analysis, as done in Righetto et al. 2012, in a spatially implicit setting). To that end, the model should be further extended to include partial/waning immunity because 
individuals recovered from waterborne diseases usually can become susceptible again after a certain time.

Despite the inevitable limitations, we believe that the generality of the mathematical approach used here to derive invasion conditions for waterborne pathogens in a spatially explicit network model subject to seasonal fluctuations could possibly be applied to other diseases (not necessarily waterborne) as well as to other geographic settings (not necessarily river networks). We thus suggest that our results, general as they are because they reduce exactly to particular cases already dealt with for spatially homogeneous and/or time-invariant conditions, may define a framework (as opposed to a model) for the realistic description of waterborne pathogen invasion and the geography of epidemic spread addressed by spatially explicit, multilayered and time-varying network models.

Acknowledgments The authors wish to thank two anonymous referees for their helpful comments on the manuscript. LM, EB and AR acknowledge the support provided by ERC advanced grant program through the project RINEC-227612 and by the SFN/FNS projects 200021_124930/1 and CR2312_138104/1. MG and AR acknowledge the support from the SFN/FNS project IZK0Z2_139537/1 for international cooperation.

\section{References}

Akanda AS, Jutla S, Islam S (2009) Dual peak cholera transmission in Bengal Delta: a hydroclimatological explanation. Geophys Res Lett 36:L19401

Altizer S, Dobson A, Hosseini P, Hudson P, Pascual M, Rohani P (2006) Seasonality and the dynamics of infectious diseases. Ecol Lett 9:467-484

Bacaër N (2007) Approximation of the basic reproduction number $R_{0}$ for vector-borne diseases with a periodic vector population. Bull Math Biol 69:1067-1091

Bacaër N, Ait Dads E (2012) On the biological interpretation of a definition for the parameter $R_{0}$ in periodic population models. J Math Biol 65:601-621

Bacaër N, Gomes MGM (2009) On the final size of epidemics with seasonality. Bull Math Biol 71:1954-1966

Bacaër N, Guernaoui S (2006) The epidemic threshold of vector-borne diseases with seasonality. J Math Biol 53:421-436

Bani-Yaghoub M, Gautam R, Shuai Z, van den Driessche P (2012) Reproduction numbers for infections with free-living pathogens growing in the environment. J Biol Dyn 6:923-940

Bertuzzo E, Maritan A, Gatto M, Rodriguez-Iturbe I, Rinaldo A (2007) River networks and ecological corridors: reactive transport on fractals, migration fronts, hydrochory. Water Resour Res 43:W04419

Bertuzzo E, Gatto M, Maritan A, Azaele S, Rodriguez-Iturbe I, Rinaldo A (2008) On the space-time evolution of a cholera epidemic. Water Resour Res 44:W01424

Bertuzzo E, Casagrandi R, Gatto M, Rodriguez-Iturbe I, Rinaldo A (2010) On spatially explicit models of cholera epidemics. J R Soc Interface 7:321-333

Bertuzzo E, Mari L, Righetto L, Gatto M, Casagrandi R, RodriguezIturbe I, Rinaldo A (2012) Hydroclimatology of dual-peak cholera epidemics: inferences from a spatially explicit model. Geophys Res Lett 39:L05403
Bittanti S, Colaneri P (2009) Periodic systems, filtering and control. Springer, London

Bouma MJ, Pascual M (2001) Seasonal and interannual cycles of endemic cholera in Bengal 1891-1940 in relation to climate and geography. Hydrobiologia 460:147-156

Capasso V, Paveri-Fontana SL (1979) A mathematical model for the 1973 cholera epidemic in the European Mediterranean Region. Rev Epidemiol Santé Publique 27:121-132

Casagrandi R, Gatto M (2006) The intermediate dispersal principle in spatially explicit metapopulations. J Theor Biol 239:22-32

Codeço CT (2001) Endemic and epidemic dynamics of cholera: the role of the aquatic reservoir. BMC Infect Dis 1:1

Colwell RR (1996) Global climate and infectious disease: the cholera paradigm. Science 274:2025-2031

de Magny GC, Murtugudde R, Sapiano MRP, Nizam A, Brown CW, Busalacchi AJ, Yunus M, Mohammad Nair GB, Gil AI, Lanata CF, Calkins J, Manna B, Rajendran K, Bhattacharya MK, Huq A, Sack RB, Colwell RR (2008) Environmental signatures associated with cholera epidemics. Proc Natl Acad Sci USA 105:17676-17681

Diekmann O, Heesterbeek JAP (2000) Mathematical epidemiology of infectious diseases: model building, analysis and interpretation. Wiley, Chichester

Diekmann O, Heesterbeek JAP, Metz JAJ (1990) On the definition and the computation of the basic reproduction ratio $R_{0}$ in models for infectious diseases in heterogeneous populations. J Math Biol $28: 365-382$

Diekmann O, Heesterbeek JAP, Roberts MG (2010) The construction of next-generation matrices for compartmental epidemic models. J R Soc Interface 7:873-885

Eisenberg JNS, Desai MA, Levy K, Bates SJ, Liang S, Naumoff K, Scott JC (2007) Environmental determinants of infectious disease: a framework for tracking causal links and guiding public health research. Environ Health Perspect 115:1216-1223

Eisenberg MC, Kujbida G, Tuite AR, Fisman DN, Tien JH (2013a) Examining rainfall and cholera dynamics in Haiti using statistical and dynamic modeling approaches. Epidemics 5:197-207

Eisenberg MC, Shuai Z, Tien JH, van den Driessche P (2013b) A cholera model in a patchy environment with water and human movement. Math Biosci 246:105-112

Emch M, Yunus M, Escamilla V, Feldacker C, Ali M (2010) Local population and regional environmental drivers of cholera in Bangladesh. Environ Health 9:2

Erlander S, Stewart NF (1990) The gravity model in transportation analysis-Theory and extensions. VSP Books, Zeist

Ferrière R, Gatto M (1995) Lyapunov exponents and the mathematics of invasion in oscillatory or chaotic populations. Theor Popul Biol 48:126-171

Gatto M, Mari L, Bertuzzo E, Casagrandi R, Righetto L, RodriguezIturbe I, Rinaldo A (2012) Generalized reproduction numbers and the prediction of patterns in waterborne disease. Proc Natl Acad Sci USA 48:19703-19708

Gatto M, Mari L, Bertuzzo E, Casagrandi R, Righetto L, RodriguezIturbe I, Rinaldo A (2013) Spatially explicit conditions for waterborne pathogen invasion. Am Nat 182:328-346

Gaudart J, Rebaudet S, Barrais R, Boncy J, Faucher B, Piarroux M, Magloire R, Thimothe G, Piarroux R (2013) Spatio-temporal dynamics of cholera during the first year of the epidemic in Haiti. PLoS Negl Trop Dis 7:e2145

Guerrant RL (1986) The global problem of amebiasis: current status, research needs, and opportunities for progress. Rev Infect Dis $8: 218-227$

Harris TE (1974) Contact interactions on a lattice. Ann Probab 2:969988

Hashizume M, Armstrong B, Hajat S, Wagatsuma Y, Faruque ASG, Hayashi T, Sack DA (2008) The effect of rainfall on the incidence of cholera in Bangladesh. Epidemiology 19:103-110 
Heesterbeek JAP, Roberts MG (1995a) Threshold quantities for helminth infections. J Math Biol 33:415-434

Heesterbeek JAP, Roberts MG (1995b) Threshold quantities for infectious diseases in periodic environments. J Biol Syst 4:779-787

Johnson AR, Hatfield CA, Milne BT (1995) Simulated diffusion dynamics in river networks. Ecol Model 83:311-325

Klausmeier CA (2008) Floquet theory: a useful tool for understanding nonequilibrium dynamics. Theor Ecol 1:153-161

Lipp EK, Huq A, Colwell RR (2002) Effects of global climate on infectious disease: the cholera model. Clin Microbiol Rev 15:757770

Mari L, Bertuzzo E, Casagrandi R, Gatto M, Levin SA, RodriguezIturbe I, Rinaldo A (2011) Hydrologic controls and anthropogenic drivers of the zebra mussel invasion of the Mississippi-Missouri river system. Water Resour Res 47:W03523

Mari L, Bertuzzo E, Righetto L, Casagrandi R, Gatto M, RodriguezIturbe I, Rinaldo A (2012a) Modelling cholera epidemics: the role of waterways, human mobility and sanitation. J R Soc Interface 9:376-388

Mari L, Bertuzzo E, Righetto L, Casagrandi R, Gatto M, RodriguezIturbe I, Rinaldo A (2012b) On the role of human mobility in the spread of cholera epidemics: towards an epidemiological movement ecology. Ecohydrology 5:531-540

Mari L, Casagrandi R, Bertuzzo E, Rinaldo A, Gatto M (2014) Metapopulation persistence and species spread in river networks. Ecol Lett 17:426-434

McMichael AJ, Woodruff RE, Hales S (2006) Climate change and human health: present and future risks. Lancet 367:859-869

Mendelsohn J, Dawson T (2008) Climate and cholera in KwaZuluNatal, South Africa: the role of environmental factors and implications for epidemic preparedness. Int $\mathrm{J}$ Hyg Environ Health 211:156-162

Newman MEJ (2005) Power laws, Pareto distributions and Zipf's law. Contemp Phys 46:323-351

Pascual M, Rodó X, Ellner SP, Colwell RR, Bouma MJ (2000) Cholera dynamics and El Niño Southern Oscillation. Science 289:17661769

Pascual M, Bouma MJ, Dobson AP (2002) Cholera and climate: revisiting the quantitative evidence. Microbes Infect 4:237-245

Pascual M, Chaves LF, Cash B, Rodó X, Yunus M (2008) Predicting endemic cholera: the role of climate variability and disease dynamics. Clim Res 36:131-140

Reiner RC, King AA, Emch M, Yunus M, Faruque ASG, Pascual M (2012) Highly localized sensitivity to climate forcing drives endemic cholera in a megacity. Proc Natl Acad Sci USA 109:2033-2036

Righetto L, Casagrandi R, Bertuzzo E, Mari L, Gatto M, RodriguezIturbe I, Rinaldo A (2012) The role of aquatic reservoir fluctuations in long-term cholera patterns. Epidemics 4:33-42

Righetto L, Bertuzzo E, Mari L, Schild E, Casagrandi R, Gatto M, Rodriguez-Iturbe I, Rinaldo A (2013) Rainfall mediations in the spreading of epidemic cholera. Adv Water Resour 60:34-46
Rinaldi S, Muratori S (1993) Conditioned chaos in seasonally perturbed predator-prey models. Ecol Model 69:79-97

Rinaldo A, Rodriguez-Iturbe I, Rigon R, Bras R, Ijjasz-Vasquez E, Marani A (1992) Minimum energy and fractal structures of drainage networks. Water Resour Res 28:2183-2195

Rinaldo A, Bertuzzo E, Mari L, Righetto L, Blokesch M, Gatto M, Casagrandi R, Murray M, Vesenbeckh S, Rodriguez-Iturbe I (2012) Reassessment of the 2010-2011 Haiti cholera outbreak and rainfall-driven multiseason projections. Proc Natl Acad Sci USA 109:6602-6607

Rinaldo A, Rigon R, Banavar JR, Maritan A, Rodriguez-Iturbe I (2014) Evolution and selection of river networks: statics, dynamics, and complexity. Proc Natl Acad Sci USA 111:24172424

Rodriguez-Iturbe I, Rinaldo A, Rigon R, Bras R, Ijjasz-Vasquez E, Marani A (1992) Fractal structures as least energy patterns-the case of river networks. Geophys Res Lett 19:889-892

Ruiz-Moreno D, Pascual M, Bouma M, Dobson A, Cash B (2007) Cholera seasonality in Madras. EcoHealth 4:52-62

Sardar T, Mukhopadhyay S, Bhowmick AR, Chattopadhyay J (2013) An optimal cost effectiveness study on Zimbabwe cholera seasonal data from 2008-2011. PLoS ONE 8:e81231

Tian JP, Wang J (2011) Global stability for cholera epidemic models. Math Biosci 232:31-41

Tien JH, Earn DJD (2010) Multiple transmission pathways and disease dynamics in a waterborne pathogen model. Bull Math Biol 72:1506-1533

Truscott J, Ferguson NM (2012) Evaluating the adequacy of gravity models as a description of human mobility for epidemic modelling. PLoS Comput Biol 8:e1002,699

Tuljapurkar S, Orzack S (1980) Population dynamics in variable environments. I. Long-run growth rates and extinction. Theor Popul Biol 18:314-342

van den Driessche P, Watmough J (2002) Reproduction numbers and sub-threshold endemic equilibria for compartmental models of disease transmission. Math Biosci 180:29-48

Wang W, Zhao XQ (2008) Threshold dynamics for compartmental epidemic models in periodic environments. J Dyn Diff Equat 20:699717

World Health Organization (2008) The global burden of disease: 2004 Update. WHO Press, Geneva

World Health Organization (2010) Prevention and control of cholera outbreaks: WHO policy and recommendations. Tech. rep. World Health Organization, Regional Office for the Eastern Mediterranean

Zhang F, Zhao XQ (2007) A periodic epidemic model in a patchy environment. J Math Anal Appl 325:496-516

Zhou X, Cui J (2013) Threshold dynamics for a cholera epidemic model with periodic transmission rate. Appl Math Model 37:3093-3101

Zipf GK (1949) Human behavior and the principle of least effort: an introduction to human ecology. Addison-Wesley, Cambridge 